\title{
Cuando la memoria también habla del porvenir. El flujo temporal en los discursos de reivindicación identitaria en Aguacatenango (Chiapas)
}

When memory also speaks of the future. Temporal flow in identity claims in Aguacatenango (Chiapas)

Quand la mémoire parle aussi de l'avenir. Le flux temporel dans les discours de revendication identitaire à Aguacatenango (Chiapas)

Marie Chosson

\section{OpenEdition}

Journals

Edición electrónica

URL: http://journals.openedition.org/jsa/15458

DOI: $10.4000 /$ jsa. 15458

ISSN: 1957-7842

Editor

Société des américanistes

Referencia electrónica

Marie Chosson, « Cuando la memoria también habla del porvenir. El flujo temporal en los discursos de reivindicación identitaria en Aguacatenango (Chiapas) », Journal de la société des américanistes [En línea], Maya times | 2017, Publicado el 31 diciembre 2017, consultado el 14 noviembre 2019. URL : http://journals.openedition.org/jsa/15458; DOI : 10.4000/jsa.15458 


\title{
Cuando la memoria también habla del porvenir. El flujo temporal en los discursos de reivindicación identitaria en Aguacatenango (Chiapas)
}

\author{
Marie CHOsson*
}

La solicitud oficial de una independencia administrativa desencadenó, en parte de la población de la localidad tseltal de Aguacatenango, un proceso de reflexiones colectivas a fin de elaborar un discurso que pudiera demostrar a las autoridades exteriores la coherencia social y la continuidad de la existencia del grupo aldeano. En esta voluntad de legitimar el ejercicio de la autoridad sobre lo que consideran como su territorio, la movilización de la memoria colectiva ocupó un lugar central en la construcción de esos discursos de reivindicación. Esta contribución se propone enfatizar una percepción particular de las temporalidades a través del análisis de la manera en que los tseltal tradicionalistas de esta localidad organizaron, en dichos discursos y según sus intenciones, las referencias entrecruzadas al tiempo largo, a la memoria vivida y a las proyecciones en el porvenir. Este análisis de las modalidades de las estrategias discursivas permitirá aclarar sus representaciones del flujo temporal y del papel concedido a los hombres en el mantenimiento de su equilibrio. [Palabras claves: temporalidades, memoria, estrategia discursiva, tradición oral, territorialidad, Tseltal, Maya, México.]

When memory also speaks of the future. Temporal flow in identity claims in Aguacatenango (Chiapas). For some of the population of the Tseltal village of Aguacatenango, the official request for administrative independence triggered a collective deliberation process aimed at drafting a document that would demonstrate to the external authorities the village group's social coherence and existential continuity. Stemming from this desire to legitimize the exercise of authority over what they considered their territory, this document stating their claims was constructed by mobilizing the collective memory. This contribution aims to highlight a particular perception of temporality, by analyzing how, in their discourse and according to their intentions, the Tseltal traditionalists of Aguacatenango organize intertwined references to long-term time, memory of life and forecasts of the future. This analysis of the modalities of discursive strategies will shed light on Tseltal representations of temporal flow and the role attributed to human beings in maintaining its balance.

* Institut national des langues et civilisations orientales, CESSMA, 65 rue des Grands Moulins, 75013 Paris [marie.chosson@inalco.fr]. 
[Key words: temporality, memory, discursive strategy, oral tradition, territoriality, Tseltal, Maya, Mexico.]

Quand la mémoire parle aussi de l'avenir. Le flux temporel dans les discours de revendication identitaire à Aguacatenango (Chiapas). La demande officielle d'une indépendance administrative a déclenché, pour une partie de la population du village tseltal d'Aguacatenango, un processus de réflexions collectives visant à l'organisation d'un discours censé démontrer aux autorités extérieures la cohérence sociale et la continuité de l'existence du groupe villageois. Dans cette volonté de légitimer l'exercice d'une autorité sur ce qu'ils considèrent comme leur territoire, ces discours revendicatifs ont été construits en mobilisant la mémoire collective. Cette contribution se propose de souligner une perception particulière des temporalités par l'analyse de la manière dont les Tseltal traditionalistes du village organisent, dans ces discours et selon leurs intentions, les références entrecroisées au temps long, à la mémoire vécue et aux projections dans l'avenir. Cette analyse des modalités de stratégies discursives permettra d'éclairer leurs représentations du flux temporel et du rôle attribué aux hommes dans le maintien de son équilibre. [Mots-clés : temporalité, mémoire, stratégie discursive, tradition orale, territorialité, Tseltal, Maya, Mexique.]

En la mayoría de los grupos mayas, las prácticas de memoria y las prácticas prospectivas se revelan a través de actos rituales o discursos que se pueden observar en la vida cotidiana ${ }^{1}$. Su observación y análisis nos dan informaciones acerca de la forma en la cual estos grupos conceptualizan no sólo el tiempo, sino también el lugar que ocupan los individuos dentro del mismo. Sin embargo, algunos eventos ofrecen la rara oportunidad de observar intercambios mucho más directos sobre la relación de los hombres con el pasado y el porvenir, y la manera en la cual ellos consideran el cambio. Este fue el caso, en el pueblo chiapaneco de Aguacatenango, de población de habla tseltal, cuando las autoridades iniciaron una solicitud oficial para obtener la condición de municipio libre, categoría administrativa de mayor autonomía, para la localidad. Para una parte de su población, este anuncio desencadenó un proceso de reflexión colectiva a fin de elaborar un discurso de reivindicación que pudiera demostrar la legitimidad y la continuidad de la existencia del grupo aldeano. Esta contribución se propone enfatizar las percepciones particulares de las temporalidades a través del análisis de la manera en que los tradicionalistas organizaron, en dichos discursos y según sus intenciones, las referencias entrecruzadas al tiempo largo, a la memoria vivida y a las proyecciones en el porvenir. Este análisis de las modalidades de las estrategias discursivas permitirá aclarar sus

1. Los resultados y análisis presentados en este artículo fueron desarrollados en el marco de los trabajos colectivos del ANR Fabriq'am "La fabrique des patrimoines : mémoires, savoirs et politique en Amérique indienne aujourd'hui” (ANR-12-CULT-005). 
representaciones del flujo temporal y del papel concedido a los hombres en el mantenimiento de su equilibrio.

\section{Pensar las fronteras: unidad administrativa y paisaje simbólico}

Como muchas localidades mayoritariamente indígenas del estado de Chiapas (México), el pueblo tseltal de Aguacatenango sufrió la reestructuración administrativa post revolucionaria de 1915. Estas localidades, reconocidas durante mucho tiempo como "repúblicas de indios" y luego como municipios de pleno derecho, perdieron entonces su libertad administrativa y pasaron bajo dependencia de otras cabeceras. Así, desde esa época, el territorio que abarca el centro del antiguo municipio de Aguacatenango está subordinado, como colonia, a la cabecera municipal de Venustiano Carranza, en su mayoría ladina y situada a más de una hora en coche del pueblo. Si los aguacatenantecos participan en las elecciones municipales de Venustiano Carranza, la ausencia y la indiferencia de los oficiales del municipio permitieron a las autoridades de costumbre mantener cierto grado de autonomía en la administración de los asuntos del pueblo. De hecho, el sistema de cargos, o jerarquía político-religiosa-organización ampliamente documentada en las investigaciones etnológicas mesoamericanas-sigue siendo la institución de ejecución del poder más efectiva y reconocida en la aldea. El sistema de cargos se organiza en una jerarquía en la cual alternan funciones administrativas y religiosas ${ }^{2}$. Aunque incorporados en el mismo sistema, los dos polos de este sistema son claramente distinguidos y representados por dos grupos, denominados mayordomos tratándose de los encargados religiosos y autoridades civiles tratándose de los encargados administrativos. La expresión "autoridades de costumbre" hará referencia, aquí, a los actores civiles del sistema de cargo.

$\mathrm{Si}$, teóricamente, cada hombre debe participar en el sistema de cargos, esta organización tradicional obliga también a los habitantes a participar en lo que llaman el sistema de cooperación, dedicado al buen mantenimiento del pueblo. Esta cooperación implica una participación efectiva a las obras de refección de la vía o de edificios públicos, pero también una contribución financiera a los gastos colectivos, recaudados, por ejemplo, para comprar el material necesario a esas obras o para la organización de varias fiestas religiosas patronales. La cooperación financiera suele ser muy pesada para la mayoría de los habitantes. Sólo para las fiestas patronales, se gastan, además de las compras personales, más de quinientos pesos al año por grupo familiar. A pesar de la importancia de la carga financiera la participación en el sistema continúa siendo importante, y varios individuos que han emigrado en la capital o incluso Estados Unidos siguen enviando su contribución.

2. Para una descripción detallada, véase Marie Chosson (2012). 
La reorganización administrativa post-revolucionaria otorgó al pueblo de Aguacatenango el estatuto de colonia de Venustiano Carranza, a la vez que otorgó el estatuto de localidades rurales, bajo la misma administración, a las pequeñas aldeas vecinas que antes de ese proceso dependían de Aguacatenango. Legalmente esas aldeas no tienen ninguna obligación de someterse a la influencia de las autoridades civiles de costumbre de Aguacatenango. Sin embargo, la mayoría de sus habitantes siguieron participando en ciertas obligaciones colectivas como las cooperaciones financieras. Mediante esa participación en el sistema de cooperación, los habitantes de las aldeas reconocen implícitamente pertenecer a la misma comunidad cuyo centro seriá Aguacatenango. A pesar de la reorganización territorial oficial, se puede por lo tanto observar una inadecuación entre las fronteras legales del territorio, reconocidas por el estado, y las fronteras simbólicas, definidas por sus habitantes como el espacio en el cual se ejerce la autoridad de los representantes del sistema de organización tradicional. Según esa misma lógica, fue precisamente cuando ciertas de esas aldeas desearon deshacerse de lo que los tradicionalistas ${ }^{3}$ consideran como sus deberes compartidos, que nacieron tensiones importantes.

\section{"Municipalizarse": una necesidad}

Las dos últimas décadas fueron el teatro de muchos cambios o trastornos que generaron conflictos territoriales y micromigraciones hacia esas aldeas. En la década de los 90, la multiplicación de las conversiones a otros referentes religiosos, permitió el asentamiento de tres nuevas congregaciones: una vinculada a los Testigos de Jehová, otra a la Iglesia Presbiteriana de México y la última, denominada Jesús El Buen Pastor, afiliada a la Iglesia Cristo Sana y Salva ${ }^{4}$ Los aproximadamente 300 conversos (lo que representa más o menos el 10\% de la población del pueblo) fueron, en esa misma década, violentamente expulsados del centro, y se instalaron en las aldeas vecinas. En esa misma época, otros grupos de personas también decidieron marcharse del centro, mayormente para emanciparse de las obligaciones del sistema de organización tradicional. La mayoría de esos micromigrantes se negaron entonces a participar en el sistema de cooperación tradicional. Este cambio importante y la emergencia de nuevos líderes, cuyas convicciones religiosas, políticas o económicas compiten con las de los tradicionalistas, reactivaron por lo tanto el debate acerca de la necesidad de una mayor independencia administrativa.

Unos acuerdos fueron firmados en 1996 entre los "que siguen la costumbre" y los nuevos conversos, obligando a los últimos a participar en el sistema de cooperación.

3. En este artículo, usaré la expresión "tradicionalistas" para referirme al grupo, mayoritario, de habitantes de Aguacatenango que se presentan como "los que siguen el camino de nuestros ancestros", o, con menor frecuencia, "los que no pierden la costumbre".

4. Datos citados por Carlos Monsiváis (2002) y Rivera Farfán y coautores (2005, p. 211). 
El fracaso de su aplicación empujó las autoridades de costumbre, dos años más tarde, a involucrarse una vez más en una solicitud oficial para la adquisición del estatuto de municipio libre, unidad administrativa de la organización territorial. El período de este trámite coincidía idealmente con la voluntad de las autoridades del gobierno chiapaneco, encabezadas por el gobernador Roberto Albores Guillén, de iniciar una política de remunicipalización ${ }^{5}$. Con la determinación de descentralizar, pero también, en un contexto de luchas de autodeterminación indígena, para frenar la creación espontánea de municipios autónomos zapatistas, el gobierno inició una serie de reformas encaminadas a la creación de nuevos municipios. El gobierno de Chiapas propuso entonces una lista de treinta y tres localidades elegibles -la mayoría de esas hipotéticas neolocalidades correspondían a antiguas divisiones administrativas de localidades indígenas. Cuarenta y dos otras localidades, incluyendo Aguacatenango, aprovecharon esta oportunidad para solicitar también su municipalización. Al final, sólo siete de las localidades propuestas en la primera lista fueron promulgadas municipios libres y los cambios de orientación política del estado dejan pocas esperanzas para una segunda ola de municipalización. Sin embargo, este fallo no disminuyó la motivación de las autoridades de costumbre de Aguacatenango, quienes siguieron multiplicando los esfuerzos para adquirir un nuevo estatuto administrativo.

Según mis interlocutores, este trámite administrativo es la oportunidad para que sean reconocidas legalmente las fronteras del territorio que ellos consideran como propias de la comunidad del pueblo. De hecho, desde hace mucho tiempo, los tradicionalistas supieron dibujar un mapa mental compartido y específico de su territorio, un paisaje que alberga en su seno todas las características básicas de su identidad. Si la trayectoria caótica de la situación administrativa de Aguacatenango y su región no parece haber sido un obstáculo a sus lógicas de apropiación del espacio, el trámite administrativo permitiría, en pocas palabras, legitimar oficialmente la existencia de fronteras, hasta la fecha simbólicas, del paisaje identitario en el cual se reconocen. La intención subyacente de las autoridades de costumbre es recuperar cierto control sobre las tierras para someter a los "nuevos" ocupantes a su autoridad y al sistema de gestión costumbrista. Como lo veremos más adelante, si estas consideraciones parecen ser sobre todo políticas o económicas, también pretenden mantener cierta continuidad temporal, esencial para la supervivencia del ser humano.

\section{"Acordarse" de la comunidad}

Desde un punto de vista legal, esta solicitud oficial no requiere ninguna presentación de evidencias históricas de la ocupación de estas tierras ni demostración

5. Sobre el tema, véanse el ensayo de Araceli Burguete Cal y Mayor (2004) y el libro dirigido por la misma y Xochitl Leyva Solano (2007). 
de la existencia de una cohesión social del grupo aldeano. Sin embargo, la voluntad de imponer las normas de conducta y el sistema de organización tradicionales al conjunto del territorio explica la orientación de los discursos que mencionaré aquí. Lo que mis interlocutores consideran aquí como la comunidad del pueblo se limita al solo grupo de los católicos de costumbre. Al margen de los procedimientos formales, organizaron y construyeron un discurso, dirigido principalmente al exterior, más concretamente a la persona de las autoridades del gobierno estatal chiapaneco, que tiende a defender la reivindicación de una unidad territorial por lo que ellos consideran como el grupo dominante y único legítimo, el de los que respetan la costumbre.

El anuncio de la posibilidad de organizar un proyecto de municipalización circuló muy rápidamente en el grupo de los tradicionalistas, desencadenando varios intercambios sobre el tema. Si decidieron contratar a un agente exterior al pueblo para redactar los trámites, resultó que los actores más solicitados por las autoridades de costumbre a fin de preparar este proyecto fueron los moletik y tatiletik $^{6}$. Estos últimos son personas, generalmente de edad avanzada, que han dejado de servir a la comunidad después de haber ocupado todos los cargos del sistema. Son considerados como los únicos poseedores del conocimiento de lo que, según la expresión de mis interlocutores, "se debe decir y hacer". Este conocimiento específico siempre se concibe como el resultado de una progresión gradual de la acumulación de experiencias durante el transcurso de una vida, dando legitimidad a la palabra y a los actos. Aunque ya no ocupan ningún cargo administrativo, esta capacidad otorga a los moletik y tatiletik una autoridad ideológica incontestable y un papel indispensable. Desempeñan la función decisiva de guardianes o cuidadores pero también de agente activo de la tradición. Estas reuniones fueron organizadas, a fin de darles la oportunidad de "recordar y transmitir" conocimientos que representarían verdaderos argumentos justificando la pertinencia de la solicitud de creación de una unidad territorial, la cual corresponde a lo que ellos consideran una unidad comunitaria.

La observación de la evolución de las pláticas y reflexiones en estas reuniones resultó ser una oportunidad excepcional para considerar directamente la

6. Sobre la diferencia entre estos estatutos en Aguacatenango, ver Marie Chosson (2012).

7. Cabe resaltar, aquí, el uso en tseltal de las raíces verbales na' y nop. En el diccionario de Marianna Slocum, Florencia Gerdel y Manuel Cruz Aguilar (1999), la raíz del verbo transitivo $n a$ ' posee los valores semánticos de pensar, saber, entender pero también de recordarse. La raíz del verbo transitivo nop remite a la acción de pensar, aprender pero también imitar, cuando está usado acompañado de la raíz o 'tan, “corazón”. Esta variabilidad podría explicarse por la interpretación de Pedro Pitarch Ramón (1996, p. 97), quien considera que entender en tseltal es "conjeturar un sentido". Esa amplitud semántica nos demuestra que la adquisición, el recuerdo y la transmisión del saber recurren principalmente a la sensibilidad de los individuos. Véase también el análisis de Aurore Monod Becquelin (1993). 
manera en la que abordaron el tema de la relación particular que mantienen con el pasado y el presente, pero también su concepción de la proyección en el futuro. Efectivamente, en un contexto donde las nociones de espacio y tiempo están ligadas de forma complementaria, varias de esas pláticas se referían a la importancia de la inscripción del grupo pueblerino en el tiempo.

\section{Compartir un mismo espacio-tiempo}

No parece raro que este tipo de reivindicación insista sobre la continuidad histórica de la existencia del grupo. Pero, en este caso, la irrupción de la problemática del tiempo en las conversaciones sobre el territorio depende de una lógica mucho más profunda. En los grupos mayas, la existencia del concepto de espacio-tiempo como principio unitario fue destacado en múltiples estudios etnológicos y etnolingüísticos, como, por ejemplo, en el análisis de la tradición oral tzotzil del pueblo de Chamula de Gary Gossen (1974). Para este autor el principio de espacio-tiempo "parece subyacer la visión del orden Chamula en el universo moral, como en la estética" ${ }^{8 "}$ (Gossen 1974, p. 29)9. Estudios más recientes, como los de Piero Gorza (2002) en Chiapas o Valentina Vapnarsky (2003) en Quintana Roo, subrayaron, por su parte, la concepción de una inscripción del tiempo y de la memoria en el espacio, en el cual se funden literalmente. De hecho, el territorio es concebido como un espacio que alberga en su seno a los actores humanos del presente, pero también a otras entidades, santos tutelares, dueños de los espacios y antepasados, que siguen actuando con los vivos. A través de sus actores, el pasado está así grabado en las particularidades geográficas a las cuales se confiere atributos espirituales.

Según mis interlocutores, no son raras las manifestaciones de esas mismas entidades. Aparecen físicamente, por vía de los sueños, o se manifiestan a través de rayos o eventos extraordinarios como un corte general de luz. Esas manifestaciones son, la mayoría del tiempo, interpretadas como una manera para esas entidades de expresar su desacuerdo con la manera de actuar de los hombres, o sus deseos de cambios. Por ejemplo, san Antonio apareció en el sueño de un hombre, para exigir que las cortinas usadas para la decoración de la iglesia en el día de su fiesta fueran verdes. Así "cambió la tradición" de decorarla de varios colores. Las interacciones entre los hombres y otras entidades parecen oportunidades ideales para asumir explícitamente transformaciones o incorporación de nuevos elementos a las prácticas percibidas como tradicionales.

8. Traducción personal del inglés: "appears to underlie the Chamula view of order in the moral universe, as in aesthetics".

9. Sobre el concepto de espacio-tiempo entre los tzotzil de Chamula, véase también el análisis del mismo autor (1974, p. 233-239). 
El territorio no es concebido únicamente como una configuración de espacios delimitados por fronteras impuestas por las instancias administrativas del estado chiapaneco, o definidos implícitamente por las diversas funciones que desempeñan: de residencia, de cultivo o salvaje. El espacio del pueblo es considerado como un cosmos en sí mismo, un espacio cuyo paisaje es vivo y dinámico y en el cual conviven múltiples tipos de entidades.

Según mis interlocutores, la comunidad del pueblo en su totalidad, abarcando a los actores humanos, pero también a otras entidades, existe y persiste porque comparte un mismo espacio-tiempo. En las pláticas, uno de los relatos de tradición oral más mencionados era la narración, común entre los mayas, del nacimiento simultáneo del territorio y del ser humano tal como existe hoy en día ${ }^{10}$. La historia de la fundación del pueblo de Aguacatenango menciona cómo, después de varias peregrinaciones, la santa patrona del pueblo, la Virgen de la Natividad, eligió instalarse en estas tierras. A su llegada, bajo sus órdenes, las piedras se pusieron en marcha y, con la ayuda de los tlacuaches, se ordenaron para edificar su casa, la iglesia, que ocuparía con sus compañeros, los otros santos tutelares del pueblo. Esta instalación constituye el indicador fundamental de un nuevo comienzo. De hecho, este evento está asociado con el nacimiento de una nueva forma de humanidad:

1) Ja' te nameixe, yalbon jmuk'ul tat, yalbon ay la winiketik namixe, ta balumile ore ili, pe ma 'ba pajal ayotik, ma' ja'uk kristiano. 2) Ben, ben pe ma' sna' ba baht, la spas wulwunel, puro bol k'op, ja 'nix ma 'yuk ya 'tel. 3) Ma' sna' ba xwe', ma'yuk sk'inal, ma'yuk skuxlejalike [...]. 4) Winikubik yu'un la stsob tal sba gente li 'i, ta olil jul te bay ore ili, och stehk'anik la ts 'in nae. 5) Ja' te Virgen Natividade la yak'beik slugar, yak'beik yixim yu'un lek kuxulik. 6) Hich och jtiempeile, como ya'yel kuxulotik li'i, como ba ayotik jo'tik. 7) Ja' hichuk ma' xch 'ay, ja' hich ay mak stup' tiempoil, ja' ya yich' kantaelbi ${ }^{11}$.

1) Los de antaño, mis abuelos me lo dijeron, me dijeron que se dice que antiguamente había hombres, en nuestra misma tierra, pero no eran igual como nosotros, no eran kristianos $^{12}$. 2) Caminaban pero sin saber adónde ir, hablaban sin cesar, puras tonterías, asimismo no tenían su trabajo. 3) No sabían adónde comer, no tenían

10. Para otros ejemplos de estos relatos de fundaciones véanse Piero Gorza (2002, p. 50-53) o Diego Méndez Guzmán (1998) para comunidades indígenas tzotziles y tseltales de Chiapas y el análisis de Valentina Vapnarsky (2001) para comunidades indígenas mayas cruso’ob de Quintana Roo.

11. Todas las citas en tseltal de este articulo proceden de entrevistas realizadas en Aguacatenango, ante varios informadores que se consideran tradicionalistas, entre 2009 y 2011.

12. En la lengua tseltal, como en otras lenguas romances, incluso el castellano, kristiano es el término usado comúnmente para designar a los humanos. No obstante, cabe mencionar una pequeña sutileza que distingue el uso tseltal. Aquí, se refiere a la humanidad nacida de la última creación, excluyendo las humanidades anteriores. 
milpas, no vivían bien [...]. 4) Se volvieron hombres cuando la gente se reunió aquí, llegaron donde está el centro de ahora, se dice que empezaron entonces a construir sus casas. 5) Es la Virgen de la Natividad que les dio su lugar, les dio su maíz para vivir bien. 6) Fue así que empezó nuestro tiempo, cómo vivimos aquí, donde nosotros estamos. 7) Es por eso que no se pierde, no se extingue el tiempo, es que está cuidado. ${ }^{13}$

Se puede observar aquí la referencia a la existencia de varios tiempos. Si todos son parte de la memoria colectiva de la tradición oral, sin embargo, se distinguen. El tiempo anterior a la fundación del pueblo es uno de los tiempos cosmológicos, el tiempo de las humanidades primitivas anteriores. La creencia en varias creaciones sucesivas es muy común entre los varios grupos mayas. Está muy bien documentada en los estudios que reportan relatos de tradición oral, como, por ejemplo, los de Gary Gossen (1974, p. 148-155) en una comunidad tzotzil de los altos de Chiapas; los de Howard Cline (1944, p. 108) con grupos lacandones de la selva chiapaneca; los de Robert Redfield y Alfonso Villa (1935, p. 331) o de Margaret Redfield (1935, p. 30) en Yucatán; o los de Morris Siegel (1943, p. 121-124) en una comunidad kanjobal de Guatemala. En este fragmento, la referencia, durante este tiempo cosmológico, a la ausencia de trabajo y cultivos agrícolas recuerda otros relatos de la rica tradición oral maya de un tiempo más oscuro. De hecho, fueron, en la mayoría de esos relatos, la inexistencia de actividad organizada y la despreocupación ritual que alentaron las malas conductas de las humanidades anteriores y les llevaron a la destrucción. Como lo destaca Gary Gossen (1974, p. 29), en la tradición oral, "los comportamientos asociales son relegados a categorías de tiempo y espacio socialmente distantes y ambiguas ${ }^{14}$ ".

Por otra parte, el tiempo de la nueva humanidad, la que sobrevive hoy, está considerado como siendo "nuestro tiempo", el mismo que el tiempo vivido contemporáneo. Es interesante ver aquí el uso de la palabra tiempo-il, préstamo del español gramaticalizada con el sufijo tseltal de sustantivo abstracto, para referirse a esa unidad de tiempo. Esa palabra se usa comúnmente en el sentido de temporada, como en la expresión stiempoil sts'une, "temporada de siembra", o stiempoil yu'un chamenae, "temporada de Semana Santa". Se refiere al concepto de una unidad de espacio-tiempo que se caracteriza por ciertos eventos o características. De esta manera, "nuestro tiempo" puede asimilarse al concepto de temporada de nuestra humanidad.

13. Todas las citas en tseltal de este articulo proceden de entrevistas realizadas en Aguacatenango, ante varios informadores que se consideran tradicionalistas, entre 2009 y 2011.

14. Traducción personal del inglés: "asocial behavior is relegated to socially distant and ambiguous categories of time and space". 


\section{Reactualizar la tradición oral}

El recuerdo de un mismo espacio-tiempo compartido por todas las entidades humanas y no humanas de la comunidad se encontró al centro de la argumentación relativa a los criterios que definen la identidad del grupo de los habitantes del pueblo. En las conversaciones sobre el cambio administrativo fueron, de hecho, mencionados muchos relatos de la tradición oral evocando las entidades no humanas, y sus interacciones con los hombres. No es sorprendente que los tradicionalistas hayan recurrido a estos relatos para legitimar sus afirmaciones. Como Robert Laughlin (1977, p. 5) lo notó también en su estudio de la tradición oral del pueblo tzotzil de Zinacantán, la narración de cuentos en el pueblo tseltal de Aguacatenango no tiene como meta principal el entretenimiento, sino el recuerdo de normas, ilustradas con lo que se considera como historias verdaderas ejemplares. Estos relatos siempre son utilizados a fines demostrativos, recordando el marco normativo de la tradición.

Las modalidades de transmisión del conocimiento tradicional requieren justamente como restricción enunciativa, entre otras cosas, el arraigo a un episodio de la experiencia inmediata. En Aguacatenango, como en el pueblo tzotzil de Chamula, donde, según Gary Gossen (1974, p. 81), "el tiempo es oportuno [para contar relatos] cuando la información en ellos es relevante o necesaria ${ }^{15}$ ", la mayoría de los relatos son contextualizados, introducidos y desarrollados en relación con acontecimientos actuales o situaciones concretas. Además de la necesidad de contextualización, Allan Burns (1983, p. 19-20) destacó, en su análisis de la categorización de las narraciones yucatecas, la importancia del carácter conversacional de múltiples tipos de discursos, incluyendo relatos. Igualmente, en Aguacatenango, la enunciación de un relato puede considerarse como un recurso que permite intercambiar dinámicamente sobre un contexto cotidiano concediendo, al mismo tiempo, según la expresión de John Haviland (1992, p. 432), gracias a su forma y a sus referencias, "un poder implícito o la impresión de veracidad" a las informaciones transmitidas. Si la evocación de tal desventura de un santo es común para ilustrar un episodio banal de la vida cotidiana, parece lógico que estos mismos relatos sean movilizados para lo que ellos consideran ser la defensa del equilibrio de la comunidad del pueblo en su totalidad. Estos relatos tienen por lo tanto como principal interés recordar las enseñanzas de los antepasados sobre las actitudes a adoptar hacia las demás entidades, que se deben satisfacer. En este sentido, este recordatorio de eventos pasados estructura el presente, ofreciendo a la comunidad humana índices de comportamientos que les permitan sobrevivir.

15. Traducción personal del inglés: "The time is right when the information in them is relevant or necessary". 
Si el pasado estructura el presente, lo contrario también es en parte cierto, gracias, en particular, al carácter maleable de la tradición oral que permite variaciones considerables. Según Robert Laughlin (1977, p. 4) "una narración zinacanteca no sólo puede cambiar drásticamente de forma de un narrador a otro, sino que el mismo narrador, en distintas ocasiones, puede intercambiar los protagonistas, reorganizar la trama e invertir la moral ${ }^{16 "}$. La plasticidad de la tradición oral permite por consiguiente la reactualización o la reinterpretación de los relatos del pasado gracias a acontecimientos recientes. La introducción de los protestantes en la narrativa de la persecución de Jesucristo por ejemplo, o la historia del pequeño san Martín que prefiere escuchar la radio en lugar de las enseñanzas de su padre, son buenos testimonios de estos fenómenos.

Esta capacidad de manipulación de la tradición oral se percibe, en el contexto de esta reivindicación, en la elección de los relatos mencionados durante estas reuniones. En el amplio repertorio de la tradición oral aguacatenanteca que había escuchado en otras ocasiones, pude observar como eligieron, manipularon y reactualizaron unos relatos, dando forma y expresión a los intereses del presente. En el marco del diseño del proyecto político-territorial, el proceso de adaptación de la tradición oral tuvo como resultado la incorporación de los nuevos actores, conversos o jóvenes que abandonan la organización tradicional, en ciertos relatos que yo había oído en otras versiones, en contextos y con intenciones muy diferentes. Por ejemplo, la historia de los dos Juan: este relato es muy común y siempre sigue una trama similar. Dos individuos, con circunstancias de nacimiento idénticas, considerados como pseudogemelos en su trayectoria inicial, eligen finalmente dos estilos de vida diferentes. Los episodios pueden variar pero la conclusión siempre es la misma: uno, en el día de su muerte, llega con éxito a la cueva donde residen los antepasados, el otro se pierde o escoge un mal camino. Este relato se enuncia frecuentemente en un contexto privado y familiar, para recordarles a los niños el peligro de las conductas que se alejan de las normas locales. Puede servir para denunciar, según las versiones, las consecuencias de la pereza humana, o del uso de la violencia. Durante las pláticas sobre el proyecto político-territorial, contaron este relato varias veces, con la variación notable que le atribuyeron al Juan perdido los defectos siempre otorgados a los protestantes. Como lo vimos antes, también fueron mencionadas, en otro relato, las consecuencias dramáticas del encuentro entre Jesucristo y los mismos protestantes, los cuales tomaron el papel habitualmente asignado a los judíos, figuras tradicionales del mal en la literatura oral.

La reactualización de esos relatos es posible gracias a la existencia de tramas o guiones típicos en los cuales, como Agnès Bergeret (2012, p. 385) lo precisa

16. Traducción personal del inglés: "not only may a Zinacantec tale change drastically from teller to teller, but the same narrator on separate occasions may switch the protagonists around, rearrange the plot, and reverse the moral". 
acerca de un corpus oral de relatos q'eqchi', "se encuentran ciertos eventos [...] y ciertas funciones de los protagonistas [...] que permitirán caracterizar cierta manera de resolver las situaciones conflictivas o contradictorias planteadas a una sociedad ${ }^{17}$ ". El uso de tramas comunes permite reponer esos relatos en la memoria colectiva, concebida como resultante de una larga transmisión. Estas prácticas de manipulación de la tradición oral facilitan la transmisión de informaciones con un menor esfuerzo cognitivo, inscribiendo las nuevas preocupaciones en el marco de un saber compartido. Además, el arraigamiento en ese marco compartido de la tradición permite legitimar las informaciones que se vehiculan.

\section{Evitar "la perdida" del tiempo: una responsabilidad humana}

La superposición del pasado con el presente nos instruye sobre la existencia de lo que no debe ser considerado como una atemporalidad fija, sino como una unidad temporal en movimiento perpetuo en la cual están conectados e interactúan, no necesariamente en orden cronológico, los acontecimientos y actores de lo que consideramos como pretérito, presente y porvenir. Esta influencia mutual atribuye, de facto, un carácter eminentemente frágil a esta unidad temporal. La posibilidad del surgimiento de un desequilibrio en esta mecánica compleja fue presentada por los participantes en las reuniones como una real amenaza:

1) Ay te mach'a ma'xch'une, ya xch'ayik costumpre, ya xch'ayik sbeil. 2) Ya stehk'an snaika ili' pe como hich xwe' hichuk chambalam, sin permiso och hulel, ma' skanta te jlumaltik, ma' skanta te lumi. 3) Ya stup'ix rasónbi. 4) Lo que pasa es que la skolta bel ta sk'ab te jtatik Jesucristo, ma'yuk mach'a ya xkoltawan... o tal vez ya xtup' 'ehuk te tiempoile. 5) Ya xhu'xaneke ya xlaj ch'ayix bel te tiempeil teme ma' jkantatik. 6) Xch'ay te scuentoil jlumaltik, ya xlajotik ehuk. 7) Yan tiempoil.

1) Hay los que no creen (o que no obedecen $)^{18}$, perdieron la costumbre, perdieron su camino. 2) Construyen sus casas aquí pero es como si comen como animales, sin permiso entraron para quedarse, no cuidan el pueblo, no cuidan la tierra. 3) Ya se extinguió la razón. 4) Lo que pasa es que Jesucristo los soltó de su mano, no hay quien los ayuda... o tal vez pueden también extinguir el tiempo. 5) Es posible también que sólo termina ya de perderse el tiempo si no lo cuidamos. 6) La historia del pueblo se perderá y moriremos también. 7) Será otro tiempo.

La posibilidad de destrucción de la humanidad actual es una creencia compartida por los diversos grupos mayas. Gary Gossen (1974, p. 24) constata: "aunque los Chamulas piensan que la cuarta creación presente es, hasta ahora,

17. Traducción personal del francés.

18. La raíz ch 'un posee los valores semánticos de creer y aceptar pero también de obedecer. 
el esfuerzo [del Creador] más exitoso, también saben que está llena de maldad y siempre en peligro de destrucción; por lo tanto están preocupados por defenderla de los comportamientos malos y de la gente malvada ${ }^{19}$ ". De la misma manera, en Aguacatenango, se piensa que si los hombres pierden sus costumbres, y no cuidan el tiempo, éste mismo puede "perderse" también. Lo que traduje aquí con la misma palabra "perder" se realiza en oraciones tseltal con dos raíces. La primera, ch'ay, tiene el significado de perder pero también de tirar, desechar o abandonar en su uso como verbo transitivo, y el significado de perderse, desaparecer o morir en su uso como verbo intransitivo. En ciertas oraciones, esta variedad semántica permite inducir una duda sobre el carácter voluntario o no de la pérdida. Por ejemplo, la expresión ya xch 'ayik sbeil en la oración 1 se refiere al acto de abandonar conscientemente su camino, implícitamente el camino de los ancestros, aunque en otro contexto podría referirse al simple hecho de perderse en el camino hacia algún lugar. La segunda raíz, tup', utilizada en el ejemplo para significar la pérdida del tiempo en la oración 5 , también es polivalente y significa, en su uso como verbo transitivo, apagar, extinguir, borrar o cancelar y, en su uso como verbo intransitivo, perderse, apagarse o extinguirse. Es comúnmente utilizada para referirse al acto de apagar algo, como en la stup' te k'ahk'e, "apagó el fuego", pero también a la cancelación de una deuda, como en tup'ix sbete, "ya se canceló su deuda", y, en nuestro ejemplo, la extinción de algo, en ya xtup' ehuk te tiempoil, "se perderá (o se extinguirá) también el tiempo". Ese tiempo compartido, nacido de la creación del pueblo y del hombre tal como existe hoy, puede así desaparecer y ser remplazado por una nueva creación, un tiempo distinto (o temporada distinta).

Esta inquietud es también el objeto principal de los discursos sobre las proyecciones hacia el futuro. Según mis interlocutores, muchas señales de alarma indican que la humanidad actual no sobrevivirá si no pone atención a las manifestaciones de desorden que podrían provocar rupturas en el equilibrio. En este caso, la pérdida de control de las tierras ocupadas por los que "han perdido su camino", representa una amenaza para la supervivencia del hombre en la medida en que priva dichas tierras de cuidados que se les debe proporcionar -es decir los rituales a las entidades que las habitan. Aunque no sea enunciado de esta manera, la función de cargador de tiempo, responsable de mantener el equilibrio del cosmos, sigue muy persistente. Como lo destaca Calixta Guiteras Holmes (1961, p. 233) en su análisis de la visión del mundo de un tzotzil, "queda a su [del hombre] discernimiento el mantener el equilibrio entre la conservación y la ruina". Para asegurar el porvenir de los hombres, es

19. Traducción personal del inglés: "Although Chamulas believe the present Fourth Creation to be his most successful effort so far, they also know that it is full of devil and ever in danger of destruction; hence, they are concerned to defend it from bad behavior and evil people". 
necesario que los hombres trabajen, día tras día, año tras año, para mantener la harmonía del tiempo, repitiendo inexorablemente las mismas negociaciones con las entidades divinas con las que cohabitan, siguiendo así "el camino de sus antepasados".

El enfoque de esos discursos en la importancia de las relaciones con las entidades divinas da la impresión de que las generaciones de hombres atraviesan el curso del tiempo sin verdaderamente marcar el paisaje histórico. De hecho, en esos relatos, no se utiliza ningún evento significativo procedente de una acción individual como marca identitaria de la constitución del grupo. Esto se explica, en parte, por la transformación sistemática de todo individuo que realiza un acto notable en figura heroica con cualidades divinas -fenómeno que, según las descripciones de Jan Vansina (1985, p. 22-24), y particularmente cuando se trata de relatos de origen, puede ser finalmente considerado como clásico en toda tradición oral. Se puede poner en paralelo esta particularidad de la historiografía local con la escasez de la memoria genealógica que no excede dos o tres generaciones.

La mayoría de los relatos que según mis interlocutores pertenecen al género de la historiología del pueblo, te scuentoil jlumaltik ${ }^{20}$, hacen referencia, como lo vimos antes, a un pasado diseñado como una categoría temporal genérica. Esta categoría abarca todo el período que sigue la fundación del pueblo y anterior a la historia de las dos o tres generaciones que preceden al locutor, aunque ocurre que los eventos relatados en estas historias sean colocados en paralelo, en un esfuerzo de contraste, con los otros pasados, los que no pertenecen más al tiempo de la humanidad. En esa misma lógica, este pasado es siempre evocado por el término general namey, "hace mucho tiempo", o puesto bajo la responsabilidad enunciativa del grupo genérico de los ancestros, los me’il tatil, "madres-padres", quienes "han dicho o dicen que..."

Esos antepasados, de los cuales es necesario seguir el camino, están reunidos en un mismo grupo categorial, el de todos los miembros humanos de la comunidad, hoy difuntos. No son identificados individualmente y son designados bajo el término genérico de jme 'tik.tatik, "nuestros padres y madres". En cuanto a su historiología, con esa concepción de un grupo genérico, los aguacatenantecos parecen proceder a una lógica parcialmente parecida a la de los jíbaros amazónicos para quienes, según Anne-Christine Taylor (1997), "hacer la historia es, en primer lugar, dedicar al olvido sus propios héroes perpetuando, en forma de arutam [entidades inmateriales], su abstracta y comunicable ejemplaridad ${ }^{21}$ ". No obstante, esos antepasados son una referencia ineludible y su mención como fuente de narración confiere un carácter de verdad indiscutible. Esta

20. Literalmente "su cuento de nuestro pueblo".

21. Traducción personal del francés. 
concepción de los antepasados recuerda las características atribuidas por los tzotziles al pasado. En su análisis de esas representaciones, Calixta Guiteras Holmes (1961, p. 251) afirma que "el pretérito se encuentra en un solo plano, sin niveles ni estratos; $y$, no obstante, quedan implícitas diferentes épocas cuando se menciona la presencia o ausencia de ciertas cosas [...]".

El uso lingüístico particular de las marcas gramaticales en cuanto a la expresión del tiempo en tseltal parece apoyar esta concepción. Según Gilles Polian (2013, p. 153) "el verbo en tseltal expresa fundamentalmente distinciones aspectuales, y no temporales. Es decir, el verbo no especifica explícitamente si la acción referida se ubica en el pasado, el presente o el futuro; [...] o bien se infiere por el contexto". Por su parte, Aurore Monod Becquelin (1993, p. 24) apunta que "es impresionante que la expresión del tiempo en cuanto al pasado, pero sobre todo al futuro, no es obligatoria en estas lenguas y una partícula del 'no ahora' puede aparecer y traducirse sin distinción al pasado o al futuro 22 ". En las narraciones movilizadas en las reuniones por la municipalización, la relativa ausencia de adverbios temporales -más frecuentes en la conversación cotidiana- que hubieran podido codificar una referencia temporal más precisa, así como la libertad de no relacionar deícticamente el contenido del relato con el tiempo de locución me parecen facilitar ese desanclaje a una temporalidad más definida que si hubiese sido caracterizada por un simple "antaño". De esa manera, la estrategia de uso de esas marcas gramaticales permite, igualmente, el énfasis en la continuidad.

La tradición oral tseltal, como muchas otras, utiliza tramas que se caracterizan por una inhibición de la individualidad humana. Esta característica tiende a esquematizar las acciones humanas, a tipificarlas para hacerlas corresponder a las formas y categorías identificadas de las experiencias de otras narraciones de la memoria colectiva. No obstante, la transparencia del hombre no debe ser interpretada como si sus acciones en el tiempo carecieran de importancia o de utilidad: sirven generalmente, como ya lo vimos antes, de lecciones, de ejemplos moralizadores sobre los comportamientos a adoptar. La ausencia de figura humana individual notable revela la prominencia de su función, indispensable pero necesariamente no individualizada, de guardián de cierta continuidad.

\section{Manejar el cambio}

Si en esos discursos la responsabilidad humana tiene por papel principal cuidar la permanencia del equilibrio del espacio-tiempo, actuando así como motor de continuidad, uno puede interrogarse sobre cómo, para los tseltal de Aguacatenango, deben ser manejados los episodios de cambio. La posibilidad

22. Traducción personal del francés. 
de la adquisición del nuevo estatuto administrativo generó varias interrogaciones sobre cambios, tales como la muy probable reconfiguración del sistema de gobierno para conformarlo con las exigencias de la lógica administrativa estatal o la llegada de fuentes de financiamiento de parte del gobierno estatal y federal. Frente a esas nuevas perspectivas de porvenir, muchos se preocuparon por las consecuencias para el hombre, positivas o negativas, y sobre todo por su adecuación al mantenimiento de lo que ellos consideran como tradición.

Las posibles consecuencias para el hombre se ponen frecuentemente en paralelo con otros tipos de cambio o experiencias concebidas como similares. Por ejemplo, en el caso preciso de la adquisición del estatuto de municipio, se hace mención de su existencia anterior.

"Antes éramos municipio. Pero se dice también que en otro tiempo éramos ricos, se dice que había oro en Las Minas. Pero vinieron a robarlo. Tal vez fue cuando dejamos de ser municipio. No sabemos si es verdad pero en este tiempo éramos ricos. Esperemos que con el regreso del municipio, tal vez la riqueza regresará23."

Este ejemplo revela una estrategia discursiva particular con respecto a la percepción del tiempo. En este enunciado se cruzan y fusionan un tiempo histórico, el de la época bastante reciente de los ascendientes del locutor cuando el pueblo era todavía municipio, y un tiempo que pertenece a una memoria colectiva más antigua, casi mítica, considerada siempre como mejor y más próspera, el tiempo de "cuando éramos ricos". Esta utopía del pasado se puede encontrar en varios otros relatos que cuentan los beneficios que los antepasados retiraban de la "fuente" de oro pero que también hacen referencias al "chisme" de la existencia de tesoros escondidos en varias partes del pueblo.

En otro ejemplo se evoca en filigrana la misma lógica de cruce de tiempos a propósito de los cambios de producción agrícola:

1) Orili' ya xhu'ix yu'un municipio, ja' mero lek xa'tehotik. 2) Pe, ma' jna' jayeb ja'wil, mero durasno li'i. 3) Tierra del durasno sbil li'i. 4) Bayel la jchontik durasno. 5) Pe ch 'ayix, ma 'yukix ja 'chuk, laj tup'kix. 6) Ja' hich aye castigo yu'un diose porque lom aragan te winike, ch'ajub te winike. 7) Ja' tulan li'to, pobreotik li'to. 8) Pe orili' lek te xa 'tehotik, lek ay, yame k'an taluke aquel tiempo, yaukme bayel jchontik.

1) Ahora es posible el municipio, trabajamos mucho mejor. 2) Pero, no sé hace cuántos años, mero durazno aquí24. 3) Su nombre de aquí es la tierra del durazno.

4) Vendíamos muchos duraznos. 5) Pero se perdieron, ya no es así, ya terminaron

23. Cita directa de una entrevista en español.

24. La antigua producción de duraznos es un tema muy recurrente en las pláticas de los aguacatenantecos. Nunca pude verificar la veracidad de esa información. Hoy en día, el único indicio que subsiste en el pueblo de este tipo de cultivo es el uso, en la toponimia, del nombre "El huerto" para designar un barrio del centro. 
extinguidos. 6) Es así que hay un castigo de Dios porque los hombres son muy haraganes, se volvieron flojos los hombres. 7) Es todavía difícil aquí, todavía somos pobres aquí. 8) Pero ahora, nuestro trabajo está bien, está bien, ya quiere venir aquel tiempo, ojalá vamos a vender mucho.

Por fin, en cuanto a los cambios debidos al desarrollo de nuevas identidades religiosas, si es cierto que están inquietos, unos de mis interlocutores costumbristas mencionaron que antes también había ciertos hombres, los judíos, que no respetaban la autoridad de Jesucristo y que igualmente habían ocupado tierras antes de ser expulsados o sumisos a ayudar a los hombres del pueblo. En esta lógica y con esta referencia, sobreentienden que su gestión para salvaguardar el territorio tendrá probablemente las mismas consecuencias para quienes no respeten las entidades divinas.

Este entrecruce de referencias al tiempo vivido individualmente, o transmitido por ascendientes directos, con el tiempo pasado de la tradición recuerda la tipificación de las experiencias humanas pero también su naturaleza cíclica. La coexistencia de concepciones lineal y cíclica del tiempo no es una particularidad maya. Como lo recuerda Nancy Farriss (1987, p. 572) "la tradición occidental, encarnada al tiempo de la Conquista española de América por la cristiandad de la última Edad Media, representa un sistema [...] que incorpora elementos cíclicos en una concepción del tiempo predominantemente lineal ${ }^{25}$ ". La especificidad maya en cuanto a la coexistencia de esas dos concepciones del tiempo consiste en una diferencia en la preeminencia de una concepción sobre la otra. Así pues la misma autora (1987, p. 573) señala que: "en el sistema de pensamiento maya [...] el tiempo lineal está incorporado en un modelo cíclico que incluye el todo ${ }^{26}$ ". La concepción de la naturaleza repetitiva de los hechos humanos es justamente lo que permite, en esos discursos, encontrar en el pasado una vasta reserva de esquemas de acción que pueden reproducirse y permiten así proyectarse en el porvenir. Para legitimar el cambio, parece necesario buscar en la memoria colectiva una experiencia con consecuencias más o menos similares a las inducidas por el cambio en cuestión. Una vez más, la flexibilidad del conocimiento llamado tradicional permite una estrategia de organización del discurso de reivindicación, que recurre de manera intencional a la memoria colectiva según las preocupaciones del momento y los deseos de proyecciones en el futuro.

Otro aspecto importante de la gramaticalización de las referencias al tiempo es la variedad de las construcciones que se refieren a acciones que podrían

25. Traducción personal del inglés: "The Western tradition, embodied at the time of the Spanish conquest of America in the late medieval Christianity, represents a system [...] that incorporates cyclical elements into a predominantly linear conception of time".

26. Ibidem. 
producirse en el porvenir ${ }^{27}$. En tseltal, no existe, tal como lo describe Valentina Vapnarsky (2009, este volumen) para el maya yucateco, una forma gramatical para la referencia a un futuro histórico y profético y "predeterminado, o más exactamente determinado por un control independiente de la intención y voluntad humanas".

De hecho ni existe una forma gramatical que sea dedicada únicamente a la expresión del futuro. Si, la mayoría del tiempo, en esos discursos, los aguacatenantecos prefieren usar una forma aspectual de incompletivo, las pocas otras construcciones dirigidas hacia el futuro insisten, con el uso del sufijo - $u k$ de irrealis o la partícula dubitativa -wan, sobre el carácter incierto no tanto del futuro sino de las acciones humanas en el porvenir.

En el uso de los recursos de la lengua tseltal, el porvenir de las acciones humanas se caracteriza por lo tanto por la incertidumbre, o por la expresión modal de un abanico de posibilidades. Al contrario, conceptualmente, las estrategias retóricas de organización del discurso revelan, en el ámbito de las comunicaciones colectivas, una predominante concepción cíclica del tiempo. Lo que parece una contradicción aquí, subraya otra vez, según mi punto de vista, la importancia de la función humana. Las decisiones del ser humano de no "perder u olvidar su camino" podrían evitar la destrucción de esa "temporada de humanidad", destrucción concebida como previsible e inevitable si el hombre no respeta "el camino de los antepasados". En su concepción de un tiempo lineal subordinado e incluido en un tiempo cíclico, no son tanto las acciones humanas que se repiten inexorablemente, sino las consecuencias de ciertas acciones. La responsabilidad de elegir "el buen camino" para evitar el castigo de Dios y asegurarse del porvenir depende de la voluntad humana.

En vez de referencias al pasado y futuro como marcas temporales cronológicas fijas, la construcción de los discursos de reivindicación revelan prácticas de memoria y prácticas prospectivas que comparten los mismos mecanismos y son asociados en el proceso cognitivo ${ }^{28}$. Si, según estudios de ciencia cognitiva, es imposible, para cualquier persona, proyectarse hacia el porvenir sin la memoria de experiencias pasadas, los tseltal amplifican esta lógica con la necesidad de registrar cualquier nueva preocupación en una referencia más amplia a un saber colectivo y tradicional, validado por los actores del pasado.

Los hombres que llevan a cabo el proceso de municipalización también diseñaron explícitamente su función en este sentido. El cambio, generado por la instalación en sus tierras de nuevos actores, en competencia con los tradicionalistas, es ahora inevitable. Por lo tanto, el discurso de los tradicionalistas

27. Para más detalles sobre las diversas expresiones del tiempo en tseltal véanse Penelope Brown (2012) y Aurore Monod Becquelin (1993, p. 23-27).

28. Véanse los trabajos de Thomas Suddendorf y Mickael Corballis (1997) y los de Cristina Atance y Daniel O’Neill (2001). 
tiende a otorgarles la responsabilidad de asegurarse de que los proyectos de transformación respeten e incluso restablezcan el equilibrio del cosmos.

1) Yanaotik ta spisil ora. 2) La la kak'be hilel ta Guatemala te hunetik namixe. 3) Orili' ya kak'be ta Venustiano. 4) Namixe ma'to ayuk carretera. 5) Ya xbotik ta be ta koktik ya xbotik. 6) Orili' ya xbotik ta camión. 7) Ay mach'a baht lom namal ta a'tel, ma'ba wokol. 8) Ja' hich yu'un xawil yanaotik ta spisil ora. Lek ay pe ja' jcuente ja' jcarga ma' xch 'ay te beil.

1) A cada hora cambiamos. 2) Se dice que antes los hombres le entregaban [al gobierno] sus documentos hasta Guatemala ${ }^{29} .3$ ) Ahora los entregan en Venustiano Carranza. 4) Antes todavía no había carretera. 5) Íbamos caminando a pie. 6) Ahora vamos en camión. 7) Hay unos que van a trabajar lejos, no hay sufrimiento. 8) Así es para que veas que cambiamos a cada rato. Está bien, pero es nuestra responsabilidad, nuestra carga no perder el camino.

En esos discursos, el proceso de cambio de situación administrativa está limitado por una obligación, la del mantenimiento de cierto grado de cohesión, lo cual no significa que este proceso de cambio no cristaliza el deseo de lograr el mejoramiento de las condiciones de vida, o incluso el regreso a un periodo de prosperidad.

Lo que se refleja, de la forma más obvia, en la mayoría de estos discursos es la aparente necesidad de mantener los flujos específicos y heterogéneos del tiempo, donde se cruzan, se mezclan y sobre todo se influyen mutuamente las acciones o actores de distintas referencias temporales. Si los relatos sobre las humanidades anteriores recuerdan episodios de ruptura, la supervivencia de la comunidad y de su territorio necesita abordar el cambio no como una misma ruptura sino como un fenómeno cuyas consecuencias deben ser adaptadas a las necesidades de una continuidad relativa. Si la memoria del pasado ofrece al hombre las herramientas necesarias a fin de considerar las posibles configuraciones del porvenir, la manipulación de esta memoria le ofrece la oportunidad de usar estrategias de adaptación que le permiten aspirar a conciliar los deseos de cambios con la necesidad de "cuidar el tiempo".

En Aguacatenango, hoy en día, no existen, como en la comunidad guatemalteca maya K'ichee' de Momostenango estudiada por Barbara Tedlock (1982), especialistas rituales designados explícitamente como guardianes del tiempo. Si, para los Altos chiapanecos, Victoria Bricker (1966) postuló la equiparación de los antiguos cargadores del tiempo con los cargos del sistema actual de funcionarios indígenas, la mayoría de los discursos de los tradicionalistas en Aguacatenango evocan, por su lado, una responsabilidad compartida por la

29. Antes de 1821, el Estado actual de Chiapas dependía de la Capitanía General de Guatemala. Este mismo año proclamó su independencia de España y se unió al Primer Imperio Mexicano. 
comunidad entera. En el cambio de estatuto administrativo, al igual que con otras transformaciones, el hombre toma por lo tanto una posición y desempeña un papel indispensable: el de cargador de tiempo que, en una concepción muy maya, combina cambio y continuidad:

1) Mero lek te municipio libre, xlok' ta lek te jlumaltik. 2) Ja' hich yu'un ma' xchamkotik ma'xlajkotikaxan. 3) Kuxulto jmamtak. 4) Ba jun beil la jta tal tul ch'ion tale pues ja' ta beel, jk'antik jo'tike sigue costumpre yu'un jme'tik jtatik tul anteriormente. 5) Porque yanaotik ma'xch'ay te jtiempoil.

1) El municipio libre es muy bueno, sale mejor nuestro pueblo. 2) Así no nos morimos, no sólo morimos. 3) Todavía tendrán vida mis nietos. 4) El camino que encontré cuando crecí pues es ahí que estarán, nosotros queremos que siga la costumbre de nuestros antepasados de antaño. 5) Porque somos otros, no se pierde nuestro tiempo.

En conclusión, pudimos ver que el proyecto político-territorial de los tradicionalistas de Aguacatenango se dirige esencialmente a la recuperación de cierto control, administrativo pero también ideológico sobre lo que ellos consideran como el territorio de su pueblo. La importancia del regreso a la conformidad de la autoridad tradicional puede explicar la voluntad de los promotores del proyecto de elaborarlo según patrones "tradicionales", tanto en sus modalidades de organización (que incluyó la participación y validación de los moletik y tatiletik, suprema autoridad ideológica para los tradicionalistas), como en la voluntad manifestada de presentarlo como una repuesta a la amenaza de perder "el tiempo".

El estudio del proceso de construcción del discurso de reivindicación permitió enfatizar una tendencia a conformarlo con otros tipos de discursos tseltales que pertenecen a géneros particulares de la tradición oral. Las estrategias discursivas elegidas subrayaron la necesidad de la inscripción del grupo pueblerino en el tiempo largo de la última creación, y el traslado parcial de la responsabilidad enunciativa al grupo de los antepasados, cuyo saber y cuya autoridad no pueden ser negados. La figura retórica más recurrente en esos discursos consiste en la mezcla, en el mismo enunciado, de diferentes referencias temporales, que tiende a recordar el carácter cíclico de los eventos. El recurso a estas estrategias enunciativas permite dar al discurso, según los usos prototípicos de ciertas formas de comunicación tseltales, una mayor fuerza ilocutiva. Esa metarepresentación de la comunicación sobre el proyecto político-territorial -cuya configuración recuerda la construcción de otro tipo de documentos administrativos de reclamación territorial, como los títulos primordiales coloniale ${ }^{30}$ - revela su inclusión en el registro más amplio del género de la historiología tseltal, el scuentoil jlumaltik, que respeta los mismos modelos de estilo.

30. Sobre los títulos primordiales véase Serge Gruzinski (1991, p. 104-148). 
En cuanto a las percepciones particulares de las temporalidades, es notable que se puedan observar, en esos discursos, unas características más ampliamente mayas como la concepción de una misma unidad de espacio-tiempo compartida con varias entidades; las referencias a un pretérito genérico, que ofrece un amplio abanico de enseñanzas; y una atención particular a la ciclicidad de algunos eventos. La necesidad de inscribir los cambios del porvenir en cierta continuidad y el énfasis en la linealidad de la función humana también les permiten a los promotores del proyecto erigirse en herederos del cargo de proteger y mantener el equilibrio de la comunidad aldeana. Finalmente, de manera consciente o no, la elección de este tipo de retórica permite a los aguacatenantecos reafirmar su afiliación a lo que Miguel León Portilla (1968) consideró como la cronovisión típicamente maya, un tipo de cosmovisión cuya esencia es el tiempo.*

* Manuscrit reçu en juin 2015, accepté pour publication en juin 2016.

Este artículo forma parte del número especial Compases y texturas del tiempo entre los mayas: lo dicho, lo escrito, lo vivido.

\section{Referencias citadas}

Atance Cristina y Daniela O’Neill

2001 “Episodic Future Thinking”, Trends in Cognition Sciences, 5 (12), p. 533-539. Bergeret Agnès

2012 La quête d'autonomie des paysans mayas-q'eqchi'de Cahabón (Guatemala), 1944-2011. Trois perspectives sur les conflits de terre et les politiques de développement agricole, thèse de doctorat en anthropologie, Université Sorbonne Nouvelle Paris 3.

BRICKER Victoria Reifler

1966 "El hombre, la carga y el camino: antiguos conceptos mayas sobre tiempo y espacio, y el sistema zinacanteco de cargos", in Evon Z. Vogt (ed.), Los Zinacantecos. Un pueblo tzotzil de los Altos de Chiapas, INI, México, p. 355-370.

Brown Penelope

2012 “Time and Space in Tzeltal: Is the Future Uphill?", Frontiers in Psychology, 3 (212), p. 1-11.

Burguete Cal y Araceli Mayor

2004 "Chiapas: nuevos municipios para espantar municipios autónomos", in Rosalva Aída Hernández, Sarela Paz y María Teresa Sierra (eds), El Estado y los indígenas en tiempos del PAN. Neoindigenismo, legalidad e identidad, CIESAS/H, Cámara de Diputados/Miguel Ángel Porrúa, México, p. 137-169.

Burguete Cal, Araceli Mayor y Xochitl Leyva Solano (eds)

2007 La remunicipalización de Chiapas. Lo político y la política en tiempos de contrainsurgencia, CIESAS/H, Cámara de Diputados/Miguel Ángel Porrúa, México.

BuRns Allan F.

1983 An Epoch of Miracles. Oral Literature of the Yucatec Maya, University of Texas Press, Austin. 
Chosson Marie

2012 "Du système de charges aux statuts de prestige individuel. Réflexions sur les frontières entre espaces de pouvoir à Aguacatenango, Chiapas", Ateliers d'anthropologie [en ligne], 37, «Frontières épaisses. Altérité et continuité en pays maya et en Mésoamérique», https://ateliers.revues.org/9217

Cline Howard

1944 "Lore and Deities of the Lacandon Indians Chiapas-Mexico", Journal of American Folklore, 57, p. 107-115.

FARRISS Nancy

1987 "Remembering the Future, Anticipating the Past: History, Time and Cosmology among the Maya of Yucatan", Comparative Studies in Society and History,

Gorza Piero 29 (3), p. 566-593.

2002 Habitar el tiempo en San Andrés Larráinzar. Paisajes indígenas de los Altos de Chiapas, Otto Editore, Turín.

Gossen Gary H.

1974 Chamulas in the World of the Sun: Time and Space in a Maya Oral Tradition, Harvard University Press Cambridge, Mass.

GRUZINSKI Serge

1991 La colonización de lo imaginario. Sociedades indígenas y occidentalización en el México español. Siglos XVI-XVIII, Fondo de Cultura Económica, México.

Guiteras Holmes Calixta

1961 Los peligros del alma. Visión del Mundo de un Tzotzil, Fondo de Cultura Económica, México-Buenos Aires.

Haviland John B.

1992 “Lenguaje ritual sin ritual”, Estudios de cultura maya, XIX, p. 427-443.

LAUGHLIN Robert M.

1977 Of Cabbages and Kings. Tales from Zinacantán, Smithsonian Contributions to Anthropology, $n^{\circ} 23$, Smithsonian Institution Press, Washington.

LEÓN-PoRTILla Miguel

1968 Tiempo y realidad en el pensamiento maya. Ensayo de acercamiento, UNAM, México.

MÉndez GuZMÁn Diego

1998 Kajkanantik, jch'ulta tiketik te leke sik te chopole / El Kajkanantik: los dioses del bien y el mal: luchas de liberación de un pueblo tzeltal, Letras Mayas Contemporáneas, tercera serie, vol. 5, INI, México.

Monod BecQuelin Aurore

1993 “Temps du récit, temps de l'oubli”, in Aurore Monod Becquelin et Antoinette Molinié, avec le concours de Danièle Dehouve (eds), Mémoire de la tradition, Société d'ethnologie, Recherches thématiques, 5, Nanterre, p. 22-51.

2000 "Polyphonie thérapeutique : une confrontation pour la guérison en tzeltal", in Aurore Monod Becquelin y Philippe Erikson (eds), Les rituels du dialogue, Société d'ethnologie, Nanterre, p. 511-553.

Monsiváis Carlos

2002 "El laicismo: notas sobre el destino (a fin de cuentas venturoso) de las libertades expresivas", Configuraciones, 3ter año (7), p. 5-23. 
Cuando la memoria también habla del porvenir

Pitarch RAmón Pedro

1996 Ch'ulel: una etnografía de las almas tzeltales, Fondo de Cultura Económica, México.

Polian Gilles

2013 Gramática del tseltal de Oxchuc, CIESAS/Publicaciones de la Casa Chata, México.

REDFIELD Margaret P.

1935 The Folk Literature of a Yucatecan Town, Contribution to American Archeology, 13 (3), Carnegie Institution, Washington.

REDFIELD Robert y Alfonso Villa

1934 Chan Kom. A Maya Village, Carnegie Institution, $n^{\circ} 448$, Washington.

Rivera Farfán Carolina, María García Aguilar, Miguel Lisbona Guillén, Irene SÁnchez y Salvador MEZa Díaz

2005 Diversidad religiosa y conflicto en Chiapas. Intereses, utopias y realidades, CIESAS/UNAM/Secretaría de gobierno del estado de Chiapas/COCyTECH, México.

SIEGEL Morris

1943 "The Creation Myth and Acculturation in Acatan, Guatemala", Journal of American Folklore, 56, p. 120-126.

Slocum Marianna, Florencia Gerdel y Manuel Cruz Aguilar

1999 Diccionario Tzeltal de Bachajón, Instituto Lingüístico de Verano, Chiapas. México.

Suddendorf Thomas y Mickael C. Corballis

1997 "Mental Time Travel and the Evolution of the Human Mind", Genetic, Social, and General Psychology Monographs, 123 (2), p. 133-167.

TAYLOR Anne-Christine

1997 “L'oubli des morts et la mémoire des meurtres", Terrain [en ligne], 29, http:// terrain.revues.org/3234; DOI: 10.4000/terrain.3234.

TeDLock Barbara

1982 Time and the Highland Maya, University of New Mexico Press, Albuquerque. VANSINA Jan

1985 Oral Tradition as History, The University of Wisconsin Press, Madison.

VAPNARSKY Valentina

2001 "Cambio y continuidad en las concepciones históricas mayas: estrategias discursivas y construcción de la memoria colectiva entre los mayas macehuales de Quintana Roo", in Hostettler Ueli y Matthew Restall (eds), Maya Survivalism, Verlag Anton Saurwein, Markt Schwaben, Germany, p. 175-190.

2003 "Recorridos instauradores: configuración y apropriación del espacio y del tiempo entre los mayas yucatecos", in Alain Breton, Aurore Monod Becquelin y Mario Humberto Ruz (eds), Los espacios mayas: representaciones, utilizaciones y creencias, UNAM, IIFL, Centro de Estudios Mayas y CEMCA, México/París, p. 363-383.

2009 "Predicción y performatividad en la memoria histórica maya yucateca", in Antje Gunsenheimer, Tsubasa Okoshi Harada, John F. Chuchiak (eds), Texto y contexto: La literatura Maya Yucateca en Perspectiva Diacrónica. Estudios de Americanistas de Bonn, Shaker, Frankfurt, p. 160-189. 
